\title{
Universal duality in a Luttinger liquid coupled to a generic environment
}

\author{
Igor V. Yurkevich \\ Nonlinearity and Complexity Research Group, Aston University, Birmingham B4 7ET, United Kingdom
}

\author{
Oleg M. Yevtushenko \\ Ludwig Maximilians University, Arnold Sommerfeld Center and Center for Nano-Science, Munich, DE-80333, Germany
}

(Received 18 March 2014; revised manuscript received 23 July 2014; published 9 September 2014)

\begin{abstract}
We study a Luttinger liquid (LL) coupled to a generic environment consisting of bosonic modes with arbitrary density-density and current-current interactions. The LL can be either in the conducting phase and perturbed by a weak scatterer or in the insulating phase and perturbed by a weak link. The environment modes can also be scattered by the imperfection in the system with arbitrary transmission and reflection amplitudes. We present a general method of calculating correlation functions under the presence of the environment and prove the duality of exponents describing the scaling of the weak scatterer and of the weak link. This duality holds true for a broad class of models and is sensitive to neither interaction nor environmental modes details, thus it shows up as the universal property. It ensures that the environment cannot generate new stable fixed points of the renormalization group flow. Thus, the LL always flows toward either conducting or insulating phase. Phases are separated by a sharp boundary which is shifted by the influence of the environment. Our results are relevant, for example, for low-energy transport in (i) an interacting quantum wire or a carbon nanotube where the electrons are coupled to the acoustic phonons scattered by the lattice defect; (ii) a mixture of interacting fermionic and bosonic cold atoms where the bosonic modes are scattered due to an abrupt local change of the interaction; (iii) mesoscopic electric circuits.
\end{abstract}

The Luttinger liquid (LL) is the canonical model which describes low-energy properties of low-dimensional interacting systems [1-3]. Its applicability is amazingly broad (see the book [4] for a review) and ranges from quantum wires and carbon nanotubes [5], to fractional and spin quantum Hall systems [6-9], to mesoscopic electric circuits [10,11], to name a few. Recent increasing interest in the LLs has been stimulated by the theoretical progress in understanding physics of cold gases [12] and topological insulators [13] where the LL again plays the role of one the basic models $[14,15]$. Quasiparticles in the LL are collective waves, or plasmons, described by the universal low-energy theory which can be derived for fermionic [16] and for bosonic [17] interacting systems. It yields a power-law decay of correlation functions which have been detected experimentally via conductance measurements and a scanning tunneling microscopy both in carbon nanotubes [18-20] and quantum nanowires [21-24]. The correlation functions, in their turn, govern scaling behavior of different perturbations, applied to the LL, and related physical observables. For example, low-energy transport in the LL is extremely sensitive to imperfections. Two archetypal cases are usually considered: (i) the ideal LL, i.e., perfectly conducting phase (CP), can be perturbed by a short-range weak scatterer (WS); (ii) two ideal LLs on disjoint left/right half-axes, i.e., insulating phase (IP), can be connected via a weak-tunneling contact: the weak-link (WL) perturbation. Both perturbations scale with changing the smallest energy in the system, which can be temperature or bias. Their scaling exponents $\Delta_{\mathrm{WS}}$ and $\Delta_{\mathrm{WL}}$ are system dependent [25-31], however, they are related by the universal formula

$$
\Delta_{\mathrm{WS}} \times \Delta_{\mathrm{WL}}=1 .
$$

Equation (1) is often referred to as the duality relation between the WS and the WL. It was shown [25] that Eq. (1) follows from the duality of fields whose correlation functions yield $\Delta_{\mathrm{WS}}$ and $\Delta_{\mathrm{WL}}$. The perturbation is relevant (irrelevant) if its scaling dimension is smaller (greater) than Euclidean space dimension $d ; d=1$ for a local perturbation. Therefore, the duality (1) asserts that only one of these two perturbations is relevant, the second one being irrelevant. Reformulating in the renormalization group (RG) language, only one phase, either the CP or the IP, corresponds to the stable fixed point of the RG flow, the second phase is unstable. This immediately explains transport properties of the system: if the WS is irrelevant and the WL is relevant, the system is driven to the $\mathrm{CP}$ and it is driven to the IP in the opposite case. The duality relation has been demonstrated for a single LL not coupled to anything else, for example, surrounding bath or environment. Without environment attractive fermions and repulsive bosons fall into the $\mathrm{CP}$ limit while repulsive fermions to the IP one.

The natural question is whether the duality is robust and survives in more complex systems, like the LL coupled via the density-density interaction to a single massless bosonic mode, e.g., to the mode of acoustic phonons [32-34]. Surprisingly, elaborated and rather lengthy calculations have shown that the duality (1) holds true for an arbitrary set of systems parameters, including the reflection amplitude of the phonon mode from the imperfection [35]. It has been assumed that the phonon sees the imperfection as an elastic pointlike scatterer. Unfortunately, the cumbersome calculus did not allow the authors to identify the origin of the duality. The breakthrough has been achieved by one of us in the recent paper [36]. By using a new method, $N$ coupled LLs have been considered for the case of the generic intermode interactions which may include density-density and/or current-current channels. For $N_{c}$ conducting and $N_{i}=N-N_{c}$ insulating phases, a universal description of the Green's functions has been obtained which has manifested the duality of two sets of 

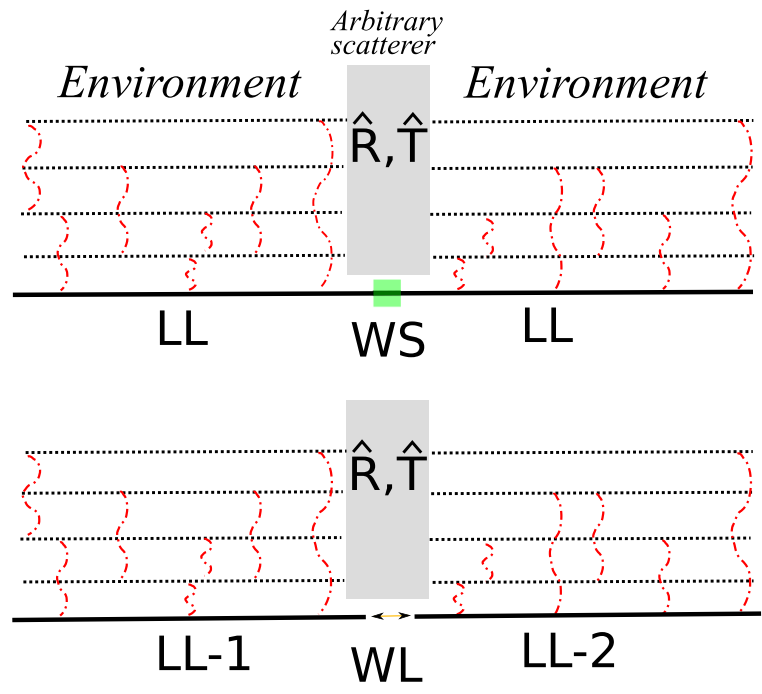

FIG. 1. (Color online) The system under consideration: the environment consists of an arbitrary number of bosonic channels (dotted lines) which interact (red wavy lines) with each other and with the Luttinger liquid; the interchannel interaction is also implied though not drawn. The bosonic modes can be scattered by the imperfection (shadowed box) described by the arbitrary scattering/transmission amplitudes $\hat{R}, \hat{T}$. The LL is either in the conducting phase (upper panel) and is perturbed by a weak scatterer (green box), or it is in the insulating phase (lower panel) and is perturbed by a weak tunneling (arrows connecting two half-axes).

the local correlation functions under interchanging $\mathrm{CP}$ and IP subsystems. This explains the origin of the duality discovered in Ref. [35] in the cases of freely propagating and fully reflected phonons. In spite of a noticeable progress, only several particular cases were considered in $[35,36]$ and the answers were obtained after lengthy algebra. For instance, only fully reflected or freely propagating scatterers were addressed in Ref. [35]; transport at small but finite energy would not allow the classification of the subsystems onto the CPs and the IPs and it is beyond the scope of Ref. [36]. Therefore, a complete explanation of the duality origin and a clear understanding of its applicability were still missing.

In this paper, we present an improved version of the analytical method which allows us to remove all listed above restrictions of the previous studies and to clearly formulate the class of systems where the duality of the given scaling exponents is present. The system which we consider consists of the LL coupled to $N-1$ massless modes of the environment (see a sketch on Fig. 1). The LL is in one of the phases, either the CP, the upper panel of Fig. 1, or the IP, the lower panel of Fig. 1. The intrachannel and interchannel interactions are arbitrary: density-density and/or the current-current interactions between all modes and channels. The current and the density are proportional to the derivatives of the chiral bosonic fields and, therefore, the effective bosonized action is quadratic. The imperfection, located at the point $x=0$, is capable of driving the LL from the WS to the WL setup and it may cause an arbitrary single-particle scattering of the environmental modes. The only assumption on the environmental modes scattering is that they can be described within quadratic theory in the bosonic degrees of freedom with proper boundary conditions at $x=0$. Particles forming LL are assumed to be of a different nature from those providing environmental bath. Therefore, local scatterer enables transitions within LL and within the environment but not mixing these two subspaces. Without loss of generality, we assume the inversion (left $\leftrightarrow$ right) symmetry of translational-invariant system and the reflection symmetry when a scatterer is placed at the origin.

We rigorously prove that the duality relation (1) holds true under these very general conditions. This, in particular, means that the environment is capable of neither modifying the stability of the RG fixed points for the single LL nor creating the new ones, unless multiparticle scattering is taken into account or the RG flow becomes multidimensional due to additional nonlinearities. Note that, in this paper, we consider only a single LL coupled to quadratic bath and postpone above extensions of the model for further studies.

Let us now specify the model in more details and outline the main steps of the calculations. Detailed derivations can be found in Appendices. We assume that $N$ one-dimensional (1D) channels each supporting two chiral modes (labeled by $\eta=\mathrm{R}, \mathrm{L})$ are described by the Lagrangian density

$$
L=\frac{1}{4 \pi} \boldsymbol{\Theta}^{\mathrm{T}}\left(\hat{\tau}_{3} \partial_{t}+\hat{V} \partial_{x}\right) \partial_{x} \Theta,
$$

with bosonic real fields

$$
\boldsymbol{\Theta}^{\mathrm{T}}=\left(\boldsymbol{\theta}_{\mathrm{R}}^{\mathrm{T}}, \boldsymbol{\theta}_{\mathrm{L}}^{\mathrm{T}}\right), \quad \boldsymbol{\theta}_{\eta}^{\mathrm{T}}=\left(\theta_{\eta}^{(1)}, \ldots, \theta_{\eta}^{(N)}\right),
$$

and with real time running over both Keldysh branches. Pauli matrices $\hat{\tau}_{j}$ act in chiral space, i.e., in space of right and left movers. The densities of chiral modes in the $i$ th channel are related to the chiral fields as $\rho_{\mathrm{R} / \mathrm{L}}^{(i)}= \pm \partial_{x} \theta_{\mathrm{R} / \mathrm{L}}^{(i)} / 2 \pi$. Diagonal entries of the $2 N \times 2 N$ matrix $\hat{V}$ describe chiral channels with velocities being renormalized due to the intrachannel interactions; the off-diagonal elements are strengths of interchannel interactions between chiral densities. The matrix $\hat{V}$ is obviously real and symmetric but its exact structure is not important for our purposes. We only assume the presence of inversion symmetry, i.e., the Lagrangian is invariant under transformation

$$
\boldsymbol{\Theta}(x) \rightarrow \hat{\tau}_{1} \boldsymbol{\Theta}(-x) .
$$

Note that (1) the choice of origin is irrelevant so far since we are dealing with the translational-invariant system at the moment; and (2) that inversion symmetry implies that arbitrary density-density interaction between chiral channels is reduced to density-density and current-current channel nonchiral interactions. Thus, the symmetries of the matrix $\hat{V}$ are

$$
\hat{V}=\hat{V}^{\mathrm{T}}, \quad \hat{\tau}_{1} \hat{V} \hat{\tau}_{1}=\hat{V} .
$$

Let us now add the imperfection at $x=0$. It can be naturally described by implying the boundary (matching) conditions. We choose to use the transfer matrix $\hat{\mathcal{T}}$

$$
\boldsymbol{\Theta}(+0)=\hat{\mathcal{T}} \boldsymbol{\Theta}(-0) .
$$

The transfer matrix $\hat{\mathcal{T}}$ must respect reflection symmetry, i.e., inversion symmetry around the origin, under the 
transformation (4), which requires

$$
\hat{\tau}_{1} \hat{\mathcal{T}} \hat{\tau}_{1}=\hat{\mathcal{T}}^{-1} \text {. }
$$

We use notations for the fields $\boldsymbol{\theta}_{\eta}( \pm 0)=\boldsymbol{\theta}_{\eta}(x= \pm 0, \omega)$ in the frequency domain, the latter allows one to accommodate possible energy dependence of scattering amplitudes. The transfer matrix can be written in terms of entries of the scattering matrix $\hat{S}$

$$
\left(\begin{array}{l}
\boldsymbol{\theta}_{\mathrm{R}}(+0) \\
\boldsymbol{\theta}_{\mathrm{L}}(-0)
\end{array}\right)=\hat{S}\left(\begin{array}{l}
\boldsymbol{\theta}_{\mathrm{L}}(+0) \\
\boldsymbol{\theta}_{\mathrm{R}}(-0)
\end{array}\right),
$$

which also must respect reflection symmetry and, therefore, obeys $\hat{\tau}_{1} \hat{S} \hat{\tau}_{1}=\hat{S}$ and can be parametrized with only two $N \times$ $N$ reflection and transmission matrices

$$
\hat{S}=\left(\begin{array}{ll}
\hat{R} & \hat{T} \\
\hat{T} & \hat{R}
\end{array}\right) .
$$

The transfer matrix is expressed in terms of $\hat{T}$ and $\hat{R}$ as follows:

$$
\hat{\mathcal{T}}=\left(\begin{array}{cc}
\hat{T}-\hat{R} \hat{T}^{-1} \hat{R} & \hat{R} \hat{T}^{-1} \\
-\hat{T}^{-1} \hat{R} & \hat{T}^{-1}
\end{array}\right) .
$$

In the model which we consider, the particles forming LL (the first channel) cannot be transformed into environment particles at the impurity since they all are of different nature; therefore, $\hat{R}$ and $\hat{T}$ are block diagonal:

$$
\hat{R}=\left(\begin{array}{ll}
R & 0 \\
0 & \hat{r}
\end{array}\right), \quad \hat{T}=\left(\begin{array}{ll}
T & 0 \\
0 & \hat{t}
\end{array}\right) .
$$

Here, the LL channel and the environment are described by scalars $R$ and $T$ and by $(N-1) \times(N-1)$ matrices $\hat{r}$ and $\hat{t}$, respectively.

Let us diagonalize the Lagrangian by a transformation

$$
\boldsymbol{\Theta}(x)=\hat{\mathcal{M}} \tilde{\boldsymbol{\Theta}}(x)
$$

which must (i) keep $\hat{\tau}_{3}$ invariant

$$
\hat{\mathcal{M}}^{\mathrm{T}} \hat{\tau}_{3} \hat{\mathcal{M}}=\hat{\tau}_{3}
$$

[cf. the first term in Eq. (2)]; and (ii) simultaneously diagonalize the matrix $\hat{V}$ which contains interactions

$$
\hat{v}=\hat{\mathcal{M}}^{\mathrm{T}} \hat{V} \hat{\mathcal{M}}=\operatorname{diag}\left(v_{1}, \ldots, v_{N} ; v_{1}, \ldots, v_{N}\right) .
$$

The matrix $\hat{\mathcal{M}}$ is real and belongs to the pseudo-orthogonal group $O(N, N)$ [see Eq. (13)]. We note that Eq. (13) is equivalent to the preservation of the Kac-Moody algebra in operator technique

$$
\left[\hat{\boldsymbol{\Theta}}(x), \hat{\boldsymbol{\Theta}}^{\mathrm{T}}\left(x^{\prime}\right)\right]=i \pi \operatorname{sign}\left(x-x^{\prime}\right) \hat{\tau}_{3},
$$

with $\left[\hat{\boldsymbol{\Theta}}(x), \hat{\boldsymbol{\Theta}}^{\mathrm{T}}\left(x^{\prime}\right)\right] \equiv \hat{\boldsymbol{\Theta}}(x) \otimes \hat{\boldsymbol{\Theta}}^{\mathrm{T}}\left(x^{\prime}\right)-\hat{\boldsymbol{\Theta}}\left(x^{\prime}\right) \otimes \hat{\boldsymbol{\Theta}}^{\mathrm{T}}(x)$.

In Eq. (14), we have taken into account the reflection symmetry on the initial (interacting) problem via the relation $\hat{v}=\hat{\tau}_{1} \hat{v} \hat{\tau}_{1}$ which holds true if

$$
\hat{\tau}_{1} \hat{\mathcal{M}} \hat{\tau}_{1}=\hat{\mathcal{M}}
$$

Equations (13) and (16) define the full symmetry of the transformation matrix $\hat{\mathcal{M}}$ [37].
To resolve all above-formulated symmetry restrictions, we can parametrize the transformation matrix $\hat{\mathcal{M}}$ with a single $N \times N$ matrix $\hat{M}$ :

$$
\hat{\mathcal{M}}=\frac{1-\tau_{1}}{2} \otimes \hat{M}+\frac{1+\tau_{1}}{2} \otimes\left(\hat{M}^{-1}\right)^{\mathrm{T}} .
$$

After diagonalization of the Lagrangian, one obtains the theory in terms of free noninteracting fields $\tilde{\boldsymbol{\Theta}}$ scattered by the imperfection. These free fields have a very simple Green's function $i \hat{\tilde{G}}=\left\langle\tilde{\Theta} \otimes \tilde{\Theta}^{\mathrm{T}}\right\rangle$ which can be written in the standard scattering state representation [38]. The latter requires boundary conditions for $\tilde{\boldsymbol{\Theta}}$ which are obtained after inserting Eq. (12) into (6):

$$
\tilde{\boldsymbol{\Theta}}(+0)=\hat{\tilde{\mathcal{T}}} \tilde{\boldsymbol{\Theta}}(-0), \quad \hat{\tilde{\mathcal{T}}}=\hat{\mathcal{M}}^{-1} \hat{\mathcal{T}} \hat{\mathcal{M}}
$$

Due to the symmetry (16), the new transfer matrix $\hat{\tilde{\mathcal{T}}}$ has the same structure as $\hat{\mathcal{T}}$ [cf. Eq. (10)], but with new scattering amplitudes $(\hat{\tilde{R}}, \hat{\tilde{T}})[39]$.

The Green's function of the original fields $\hat{G}$ can be found from the transformation (12):

$$
\hat{G}\left(x, x^{\prime}\right)=\hat{\mathcal{M}} \hat{\tilde{G}}\left(x, x^{\prime}\right) \hat{\mathcal{M}}^{\mathrm{T}} .
$$

The matrix $\hat{\mathcal{M}}$ depends neither on coordinates nor on time. Therefore, the relation between $\hat{G}$ and $\hat{\tilde{G}}$ is local. Equation (19) completes the formal description of the system so we have all information which is necessary to calculate the scaling dimension of the WS and of the WL.

These perturbations acting in the LL channel are described by [36]

$$
\begin{aligned}
& L_{\mathrm{WS}}=\lambda \cos [\Phi(T=1)], \\
& L_{\mathrm{WL}}=t \cos [\Phi(T=0)],
\end{aligned}
$$

where the field $\Phi$ is the difference between two (right- and left-) incoming chiral fields of the first channel

$$
\Phi(T)=\theta_{\mathrm{R}}^{(1)}(x=-0, t)-\theta_{\mathrm{L}}^{(1)}(x=+0, t) .
$$

The notation in Eqs. (20)-(22) stresses that the field $\Phi$ (and its correlation function) depends on boundary conditions and, in particular, on boundary conditions in the LL channel. In Eq. (20), we assume that the LL channel is in the CP with $T=1, R=0$ and it is perturbed by $L_{\mathrm{WS}}$. In the IP with $T=0, R=1$ the LL channel is perturbed by $L_{\mathrm{WL}}$. After integrating out high-energy degrees of freedom with the energy lying above the running cutoff $\varepsilon$, the Green's function $\mathcal{G}=-i\langle\Phi \Phi\rangle$ with retarded component

$$
\mathcal{G}=-\frac{2 \pi i}{\omega+i 0} \Delta_{11}(T)
$$

defines one-loop RG equations

$$
\frac{\partial \ln \lambda}{\partial \ln \varepsilon}=\Delta_{\mathrm{WS}}-1, \quad \frac{\partial \ln t}{\partial \ln \varepsilon}=\Delta_{\mathrm{WL}}-1,
$$

where

$$
\Delta_{\mathrm{WS}}=\Delta_{11}(T=1), \quad \Delta_{\mathrm{WL}}=\Delta_{11}(T=0) .
$$


The scattering and the tunneling amplitudes acquire an effective power-law dependence on the smallest energy scale:

$$
\lambda(\varepsilon) \sim \lambda \varepsilon^{\Delta_{\mathrm{WS}}-1}, \quad t(\varepsilon) \sim t \varepsilon^{\Delta_{\mathrm{WL}}-1} .
$$

The scaling exponent $\Delta_{11}(T)$ is found from the local Green's function at the origin and can be written as " 11 " element of the $N \times N$ matrix $\hat{\Delta}$ defined in the channel space:

$$
\hat{\Delta}(T)=\frac{1}{2} \hat{M}[1-\hat{\tilde{R}}+\hat{\tilde{T}}] \hat{M}^{\mathrm{T}}+\frac{1}{2}\left(\hat{M}^{-1}\right)^{\mathrm{T}}[1+\hat{\tilde{R}}-\hat{\tilde{T}}] \hat{M}^{-1} \text {. }
$$

Equation (25) can be reduced to [40]

$$
\hat{\Delta}(T)=\left[\hat{\xi}^{-1}+\hat{\delta}^{-1}\right]^{-1}+[\hat{\xi}+\hat{\delta}]^{-1}
$$

where

$$
\hat{\xi}=\frac{1-(\hat{R}-\hat{T})}{1+(\hat{R}-\hat{T})}, \quad \hat{\delta}=\hat{M} \hat{M}^{\mathrm{T}} .
$$

The matrix $\hat{\delta}$ depends on interaction only and defines the scaling exponent in the absence of environment. Taking into account the block-diagonal structure of the matrices $\hat{R}$ and $\hat{T}$ [Eq. (11)], the matrix $\hat{\xi}$ is also block diagonal

$$
\hat{\xi}=\left(\begin{array}{ll}
\xi & 0 \\
0 & \hat{\zeta}
\end{array}\right),
$$

where we have introduced the scalar $\xi$ and the $(N-1) \times$ $(N-1)$ matrix $\hat{\zeta}$ :

$$
\xi=\frac{1-(R-T)}{1+(R-T)}, \quad \hat{\zeta}=\frac{1-(\hat{r}-\hat{t})}{1+(\hat{r}-\hat{t})} .
$$

The scalar $\xi$ describes the phase of the LL: (i) $\xi=\infty$ for conducting phase ( $T=1, R=0$ ) perturbed by WS; and (ii) $\xi=0$ for insulating phase $(T=0, R=1)$ perturbed by WL. The matrix $\hat{\zeta}$ describes scattering of bath modes. It is arbitrary because no additional assumptions were implied, i.e., by the proper choice of the reflection and the transmission matrices we can take into account the arbitrary environmental scattering.

Finally, combining Eqs. (23)-(28) we arrive at our main result

$$
\begin{gathered}
\Delta_{\mathrm{WS}}=\lim _{\xi \rightarrow \infty} \hat{\Delta}_{11}=\left[\hat{\delta}^{-1}+\left(\begin{array}{cc}
0 & 0 \\
0 & \hat{\zeta}^{-1}
\end{array}\right)\right]_{11}^{-1}, \\
\Delta_{\mathrm{WL}}=\lim _{\xi \rightarrow 0} \hat{\Delta}_{11}=\left[\hat{\delta}+\left(\begin{array}{ll}
0 & 0 \\
0 & \hat{\zeta}
\end{array}\right)\right]_{11}^{-1} .
\end{gathered}
$$

It gives explicit expressions for scaling dimensions of operators which perturb the LL. The well-known expression for the scaling exponents in the case of a single LL can be obtained from Eqs. (30) and (31) straightforwardly [41]. The duality relation (1) directly results from the structure of matrices in Eqs. (30) and (31) [42]. The duality is universal: details of bath scattering $\hat{\zeta}$ and of interactions $\hat{\delta}$ are all irrelevant.

To conclude, we have proven that the duality between the weak-scatterer and the weak-link scaling exponents holds true in the system where the Luttinger liquid is coupled to a very generic environment. The duality guarantees the same classification of the fixed stable points of the RG flow as in the isolated LL $[25,26]$, the coupling to the environment is unable to create new stable fixed points. The duality and the structure of the RG flow are universal and very robust because (i) the duality relation is insensitive to parameters of the LL, of the environment, and of the coupling; (ii) the type of the coupling does not influence the duality, so it can be arbitrary chiral density-density interactions; and (iii) the scattering of the environmental modes by the imperfection can be arbitrary, the duality and the RG fixed points survive even opening the system when the number of coherent particles at the imperfection is not conserved (but cf. [43]). We have restricted ourselves to a system with the reflection symmetry. This assumption is only a technical simplification which can be easily removed without changing our results. The necessary condition for the duality of the scaling exponents is the Kac-Moody algebra [Eq. (15)] [or related duality of linear combinations of the chiral fields $\boldsymbol{\theta}_{\mathrm{R}} \pm \boldsymbol{\theta}_{\mathrm{L}}$ ]. It leads to the symmetry relation (13) and allows one to derive Eq. (26). Based on our results, we can define the universality class where the duality is always present due to the symmetry (13): it includes the systems where there are no (i) direct transitions between the LL and the environment at the imperfection, (ii) multiparticle scattering, (iii) additional nonlinearities except those which are related to the WS and the WL perturbations. Presence of any of these three effects could change the RG drastically, but such extensions are beyond the scope of this paper and will be considered elsewhere.

In real experiments, our model is applicable, for instance, to an interacting quantum wire or a carbon nanotube where the electrons are coupled to the acoustic phonons scattered by the lattice defect [32]. Another physical realization is a mixture of interacting fermionic and bosonic cold atoms where the bosonic modes are scattered due to an abrupt local change of the interaction [44].

O.M.Ye. acknowledges support from the DFG through SFB-TR12, and the Cluster of Excellence, Nanosystems Initiative Munich. I.V.Y. acknowledges hospitality of the Ludwig Maximilians University, Arnold Sommerfeld Center, Munich.

\section{APPENDIX A: DIAGONALIZATION OF THE LAGRANGIAN}

Let us prove that the Lagrangian (2), with the matrix $\hat{V}$ obeying symmetry relations

$$
\hat{V}^{\mathrm{T}}=\hat{V}, \quad \hat{\tau}_{1} \hat{V} \hat{\tau}_{1}=\hat{V},
$$

can be diagonalized with the help of the matrix $\hat{\mathcal{M}}$, which has the symmetries

$$
\hat{\mathcal{M}}^{\mathrm{T}} \hat{\tau}_{3} \hat{\mathcal{M}}=\hat{\tau}_{3}, \quad \hat{\tau}_{1} \hat{\mathcal{M}} \hat{\tau}_{1}=\hat{\mathcal{M}}
$$

It is convenient to introduce rotated matrices

$$
\hat{V}_{\mathrm{L}}=\hat{L} \hat{V} \hat{L}^{-1}, \quad \hat{\mathcal{M}}_{\mathrm{L}}=\hat{L} \hat{\mathcal{M}} \hat{L}^{-1},
$$

with

$$
\hat{L}=\frac{1}{\sqrt{2}}\left(\begin{array}{cc}
1 & -1 \\
1 & 1
\end{array}\right), \quad \hat{L}^{-1}=\hat{L}^{\mathrm{T}} .
$$


Having noticed that

$$
\hat{L} \hat{\tau}_{3}=\hat{\tau}_{1} \hat{L}
$$

the symmetry relations in the new basis read as

$$
\begin{aligned}
\hat{V}_{\mathrm{L}}^{\mathrm{T}} & =\hat{V}_{\mathrm{L}}, \quad \hat{\tau}_{3} \hat{V}_{\mathrm{L}} \hat{\tau}_{3}=\hat{V}_{\mathrm{L}} ; \\
\hat{\mathcal{M}}_{\mathrm{L}}^{\mathrm{T}} \hat{\tau}_{1} \hat{\mathcal{M}}_{\mathrm{L}} & =\hat{\tau}_{1}, \quad \hat{\tau}_{3} \hat{\mathcal{M}}_{\mathrm{L}} \hat{\tau}_{3}=\mathcal{M}_{\mathrm{L}} .
\end{aligned}
$$

These constraints are solved by the

$$
\begin{aligned}
\hat{V}_{\mathrm{L}} & =\operatorname{diag}\left(\hat{V}_{1}, \hat{V}_{2}\right), \quad \hat{V}_{i}^{\mathrm{T}}=\hat{V}_{i}, \quad i=1,2 \\
\hat{\mathcal{M}}_{\mathrm{L}} & =\operatorname{diag}\left[\hat{M},\left(\hat{M}^{-1}\right)^{\mathrm{T}}\right] .
\end{aligned}
$$

Now, to prove validity of Eq. (14), we will construct the matrix $\hat{\mathcal{M}}$. We repeat Eq. (14) for the convenience of readers:

$$
\hat{\mathcal{M}}^{\mathrm{T}} \hat{V} \hat{\mathcal{M}}=\hat{v}=\operatorname{diag}\left(\hat{v}_{d}, \hat{v}_{d}\right) ; \quad \hat{v}_{d} \equiv \operatorname{diag}\left(v_{1}, \ldots, v_{N}\right) .
$$

Rotating this equation by the matrix $\hat{L}$, we find

$$
\hat{\mathcal{M}}_{\mathrm{L}}^{\mathrm{T}} \hat{V}_{\mathrm{L}} \hat{\mathcal{M}}_{\mathrm{L}}=\hat{v}
$$

which, due to the block-diagonal structure of $\hat{V}_{\mathrm{L}}$ and $\hat{\mathcal{M}}_{\mathrm{L}}$, breaks into two simultaneous diagonalizations

$$
\hat{M}^{\mathrm{T}} \hat{V}_{1} \hat{M}=\hat{v}_{d}, \quad \hat{M}^{-1} \hat{V}_{2}\left(\hat{M}^{-1}\right)^{\mathrm{T}}=\hat{v}_{d} .
$$

The matrix $\hat{V}_{1}$ is symmetric [Eq. (A8)] and real (see the main text). Therefore, we can write a decomposition

$$
\hat{V}_{1}=\hat{O}_{1}^{\mathrm{T}} \hat{\lambda}_{1} \hat{O}_{1}
$$

where $\hat{O}_{1}$ is the orthogonal matrix and $\hat{\lambda}_{1}$ is the diagonal matrix of positive (to provide stability of the Luttinger liquid) eigenvalues. The first Eq. (A12) allows us to write

$$
\hat{M}=\hat{O}_{1}^{\mathrm{T}} \hat{\lambda}_{1}^{-1 / 2} \hat{\mu}, \quad \hat{\mu}^{\mathrm{T}} \hat{\mu}=\hat{v}_{d}
$$

and we can choose

$$
\hat{\mu}=\hat{O}^{\mathrm{T}}\left(\hat{v}_{d}\right)^{1 / 2}, \quad \hat{O}^{\mathrm{T}} \hat{O}=1 .
$$

We note that the orthogonal matrix $\hat{O}$ is arbitrary so far and we will find it by using the second Eq. (A12). Namely, inserting Eqs. (A14) and (A15) into the second Eq. (A12), we obtain

$$
\hat{M}^{-1} \hat{V}_{2}\left(\hat{M}^{-1}\right)^{\mathrm{T}}=\hat{\mu}^{-1} \hat{A}\left(\hat{\mu}^{-1}\right)^{\mathrm{T}}=\hat{v}_{d},
$$

where we have introduced the auxiliary real symmetric matrix

$$
\hat{A}=\hat{\lambda}_{1}^{1 / 2} \hat{O}_{1} \hat{V}_{2} \hat{O}_{1}^{\mathrm{T}} \hat{\lambda}_{1}^{1 / 2}=\hat{O}^{\mathrm{T}}\left(\hat{v}_{d}\right)^{2} \hat{O} ;
$$

the latter equality results form Eqs. (A15) and (A16). Equation (A17) fixes the matrix $\hat{O}$. Thus, we have constructed the matrix $\hat{\mathcal{M}}$ for the arbitrary matrices $\hat{V}_{1,2}$. This completes the proof.

\section{APPENDIX B: GREEN'S FUNCTIONS}

Let us consider the Green's function $\hat{\tilde{G}}\left(x, x^{\prime}, \omega\right)$ of noninteracting chiral bosons

$i \hat{\tilde{G}}=\left\langle\tilde{\boldsymbol{\Theta}} \otimes \tilde{\boldsymbol{\Theta}}^{\mathrm{T}}\right\rangle, \quad \tilde{\boldsymbol{\Theta}}^{\mathrm{T}}=\left(\tilde{\boldsymbol{\theta}}_{\mathrm{R}}^{\mathrm{T}}, \tilde{\boldsymbol{\theta}}_{\mathrm{L}}^{\mathrm{T}}\right), \quad \tilde{\boldsymbol{\theta}}_{\eta}^{\mathrm{T}}=\left(\tilde{\theta}_{\eta}^{(1)}, \ldots, \tilde{\theta}_{\eta}^{(N)}\right)$.
The Green's function without any scatterer is diagonal in the space of channels and its retarded component reads as [cf. Eq. (2) in the main text]

$$
\begin{aligned}
\hat{g}^{-1} & =\frac{1}{2 \pi}\left(\hat{\tau}_{3} \partial_{t}+\hat{v} \partial_{x}\right) \partial_{x} \Rightarrow \hat{g}\left(q ; \omega_{+}\right)=\frac{2 \pi}{\left(\hat{\tau}_{3} \omega_{+}-\hat{v} q\right) q}, \\
\omega_{+} & \equiv \omega+i 0
\end{aligned}
$$

with the Pauli matrices $\hat{\tau}_{k}$ acting in the chiral space and the diagonal $2 N \times 2 N$ matrix $\hat{v}$ being defined in Eq. (14) of the main text. Calculating the Fourier transform, we find

$$
\begin{aligned}
& \hat{g}\left(x-x^{\prime} ; \omega_{+}\right)=g_{0} e^{i \frac{\left|x-x^{\prime}\right| \omega_{+}}{\hat{v}}}\left(\begin{array}{cc}
\theta\left(x-x^{\prime}\right) & 0 \\
0 & \theta\left(x^{\prime}-x\right)
\end{array}\right) \\
& -\frac{g_{0}}{2} \hat{\tau}_{3} \operatorname{sign}\left(x-x^{\prime}\right), \quad g_{0} \equiv-\frac{2 \pi i}{\omega_{+}}
\end{aligned}
$$

where $\theta(x)$ is the step function.

Let us now add a scatterer at $x=0$, which will be described by its transmission and reflection amplitudes. In the scattering state representation, we have to introduce incoming/outgoing fields

$$
\tilde{\Theta}_{\text {out }} \equiv\left(\begin{array}{l}
\tilde{\boldsymbol{\theta}}_{\mathrm{R}}(x=+0) \\
\tilde{\boldsymbol{\theta}}_{\mathrm{L}}(x=-0)
\end{array}\right), \quad \tilde{\Theta}_{\mathrm{in}} \equiv\left(\begin{array}{l}
\tilde{\boldsymbol{\theta}}_{\mathrm{L}}(x=+0) \\
\tilde{\boldsymbol{\theta}}_{\mathrm{R}}(x=-0)
\end{array}\right)
$$

[cf. Eq. (8) in the main text]. The correlation function of these fields

$$
i \hat{G}_{a, b} \equiv\left\langle\tilde{\Theta}_{a} \otimes \tilde{\Theta}_{b}\right\rangle, \quad a, b=\text { in/out }
$$

can be introduced as usually:

(1) Correlations of "in-in" and "out-out" fields are not affected by the scatterer and, therefore,

$$
\hat{G}_{\text {in,in }}(\omega)=\hat{G}_{\text {out }, \text { out }}(\omega)=\hat{g}\left(\omega_{+}, 0\right)=\frac{g_{0}}{2} \otimes 1_{2 N \times 2 N} .
$$

(2) Incoming fields are independent on the outgoing ones, i.e., they are not correlated:

$$
\hat{G}_{\text {in }, \text { out }}(\omega)=0 .
$$

(3) Correlations of outgoing fields with incoming ones are given by the scattering matrix

$$
\hat{G}_{\text {out }, \text { in }}(\omega)=g_{0} \hat{\tilde{S}}, \quad \hat{\tilde{S}}=\left(\begin{array}{cc}
\hat{\tilde{R}} & \hat{\tilde{T}} \\
\hat{\tilde{T}} & \hat{\tilde{R}}
\end{array}\right)
$$

[see Eq. (9) in the main text]; here, $N \times N$ matrices $\hat{T}$ and $\hat{R}$ are transmission and reflection amplitudes, respectively, and we have taken into account the symmetry of the scattering matrix with respect to the matrix $\hat{\tau}_{1}$ due to the reflection symmetry.

Combining Eqs. (B6)-(B8), we determine the Green's function $\hat{\tilde{G}}\left(x, x^{\prime} \rightarrow 0\right)$ :

$$
\begin{aligned}
& \hat{\tilde{G}}_{++} \equiv \hat{\tilde{G}}\left(x \rightarrow+0, x^{\prime} \rightarrow+0\right)=g_{0}\left(\begin{array}{cc}
1 / 2 & \hat{\tilde{R}} \\
0 & 1 / 2
\end{array}\right), \\
& \hat{\tilde{G}}_{+-} \equiv \hat{\tilde{G}}\left(x \rightarrow+0, x^{\prime} \rightarrow-0\right)=g_{0}\left(\begin{array}{ll}
\hat{\tilde{T}} & 0 \\
0 & 0
\end{array}\right),
\end{aligned}
$$




$$
\hat{\tilde{G}}_{-+} \equiv \hat{\tilde{G}}\left(x \rightarrow-0, x^{\prime} \rightarrow+0\right)=g_{0}\left(\begin{array}{cc}
0 & 0 \\
0 & \hat{\tilde{T}}
\end{array}\right), \quad(\mathrm{B} 11) \quad \begin{gathered}
\text { The Green's function of the original chiral modes } \\
i \hat{G}=\left\langle\boldsymbol{\Theta} \otimes \boldsymbol{\Theta}^{\mathrm{T}}\right\rangle, \boldsymbol{\Theta}^{\mathrm{T}}=\left(\boldsymbol{\theta}_{\mathrm{R}}^{\mathrm{T}}, \boldsymbol{\theta}_{\mathrm{L}}^{\mathrm{T}}\right), \boldsymbol{\theta}_{\eta}^{\mathrm{T}}=\left(\theta_{\eta}^{(1)}, \ldots, \theta_{\eta}^{(N)}\right)
\end{gathered}
$$

$\hat{\tilde{G}}_{--} \equiv \hat{\tilde{G}}\left(x \rightarrow-0, x^{\prime} \rightarrow-0\right)=g_{0}\left(\begin{array}{cc}1 / 2 & 0 \\ \hat{\tilde{R}} & 1 / 2\end{array}\right)$.

(B12) is related to $\hat{\tilde{G}}$ as

$$
\hat{G}\left(x, x^{\prime}\right)=\hat{\mathcal{M}} \hat{\tilde{G}}\left(x, x^{\prime}\right) \hat{\mathcal{M}}^{\mathrm{T}}
$$

[see Eq. (19) in the main text]. $\hat{G}\left(x, x^{\prime}\right)$ is needed at $x, x^{\prime}= \pm 0$ only, thus we introduce $\hat{G}_{ \pm \pm} \equiv \hat{G}\left(x= \pm 0, x^{\prime}= \pm 0\right)$ and express it in terms of $\hat{\tilde{G}}_{ \pm \pm}$:

$$
\left(\begin{array}{ll}
\hat{G}_{++} & \hat{G}_{+-} \\
\hat{G}_{-+} & \hat{G}_{--}
\end{array}\right)=\left(\begin{array}{ll}
\mathcal{M} \hat{\tilde{G}}_{++} \mathcal{M}^{\mathrm{T}} & \mathcal{M} \hat{\tilde{G}}_{+-} \mathcal{M}^{\mathrm{T}} \\
\mathcal{M} \hat{\tilde{G}}_{-+} \mathcal{M}^{\mathrm{T}} & \mathcal{M} \hat{\tilde{G}}_{--} \mathcal{M}^{\mathrm{T}}
\end{array}\right)=g_{0}\left(\begin{array}{cc}
\mathcal{M}\left(\begin{array}{cc}
1 / 2 & \hat{\tilde{R}} \\
0 & 1 / 2
\end{array}\right) \mathcal{M}^{\mathrm{T}} & \mathcal{M}\left(\begin{array}{cc}
\hat{\tilde{T}} & 0 \\
0 & 0
\end{array}\right) \mathcal{M}^{\mathrm{T}} \\
\mathcal{M}\left(\begin{array}{ll}
0 & 0 \\
0 & \hat{\tilde{T}}
\end{array}\right) \mathcal{M}^{\mathrm{T}} & \mathcal{M}\left(\begin{array}{cc}
1 / 2 & 0 \\
\hat{\tilde{R}} & 1 / 2
\end{array}\right) \mathcal{M}^{\mathrm{T}}
\end{array}\right) .
$$

\section{APPENDIX C: TRANSFER MATRIX AND SCATTERING AMPLITUDES}

Right $\leftrightarrow$ left (inversion) symmetry of the system requires the following symmetry properties of the scattering and transfer matrices:

$$
\hat{\mathcal{S}}=\hat{\tau}_{1} \hat{\mathcal{S}} \hat{\tau}_{1} \quad \text { and } \quad \hat{\tau}_{1} \hat{\mathcal{T}} \hat{\tau}_{1}=\hat{\mathcal{T}}^{-1}
$$

The first symmetry relation means

$$
\hat{\mathcal{S}}_{11}=\hat{\mathcal{S}}_{22}, \quad \hat{\mathcal{S}}_{12}=\hat{\mathcal{S}}_{21},
$$

i.e., $\hat{\mathcal{S}}$ can be parametrized with only two $N \times N$ reflection and transmission matrices:

$$
\hat{S}=\left(\begin{array}{ll}
\hat{R} & \hat{T} \\
\hat{T} & \hat{R}
\end{array}\right) .
$$

The second symmetry relation results in

$$
\left(\begin{array}{cc}
\hat{\mathcal{T}}_{22} \hat{\mathcal{T}}_{11}+\hat{\mathcal{T}}_{21}^{2} & \hat{\mathcal{T}}_{22} \hat{\mathcal{T}}_{12}+\hat{\mathcal{T}}_{21} \hat{\mathcal{T}}_{22} \\
\hat{\mathcal{T}}_{11} \hat{\mathcal{T}}_{21}+\hat{\mathcal{T}}_{12} \hat{\mathcal{T}}_{11} & \hat{\mathcal{T}}_{11} \hat{\mathcal{T}}_{22}+\hat{\mathcal{T}}_{12}^{2}
\end{array}\right)=1 .
$$

Thus, $\hat{\mathcal{T}}$ can be parametrized, for example, by elements $\hat{\mathcal{T}}_{11}$ and $\hat{\mathcal{T}}_{22}$ :

$$
\hat{\mathcal{T}}=\left(\begin{array}{cc}
\hat{\mathcal{T}}_{11} & \sqrt{1-\hat{\mathcal{T}}_{11} \hat{\mathcal{T}}_{22}} \\
-\sqrt{1-\hat{\mathcal{T}}_{22} \hat{\mathcal{T}}_{11}} & \hat{\mathcal{T}}_{22}
\end{array}\right) .
$$

Using definitions of the scattering and transfer matrices [Eqs. (6) and (8) in the main text], and excluding $\hat{\mathcal{T}}_{21}$ with the help of Eq. (C3), one can find the relation between scattering and transfer matrices:

$$
\begin{aligned}
\hat{\mathcal{S}} & =\left(\begin{array}{cc}
\hat{\mathcal{T}}_{12} \hat{\mathcal{T}}_{22}^{-1} & \hat{\mathcal{T}}_{11}-\hat{\mathcal{T}}_{12} \hat{\mathcal{T}}_{22}^{-1} \hat{\mathcal{T}}_{21} \\
\hat{\mathcal{T}}_{22}^{-1} & -\hat{\mathcal{T}}_{22}^{-1} \hat{\mathcal{T}}_{21}
\end{array}\right) \\
& =\left(\begin{array}{cc}
\hat{\mathcal{T}}_{12} \hat{\mathcal{T}}_{22}^{-1} & \hat{\mathcal{T}}_{22}^{-1} \\
\hat{\mathcal{T}}_{22}^{-1} & \hat{\mathcal{T}}_{12} \hat{\mathcal{T}}_{22}^{-1}
\end{array}\right)=\left(\begin{array}{ll}
\hat{R} & \hat{T} \\
\hat{T} & \hat{R}
\end{array}\right) .
\end{aligned}
$$

Therefore, $\hat{\mathcal{T}}_{22}=\hat{T}^{-1}$ and $\hat{\mathcal{T}}_{12}=\hat{R} \hat{T}^{-1}$ and, using again Eq. (C3), we arrive at

$$
\hat{\mathcal{T}}=\left(\begin{array}{cc}
\hat{T}-\hat{R} \hat{T}^{-1} \hat{R} & \hat{R} \hat{T}^{-1} \\
-\hat{T}^{-1} \hat{R} & \hat{T}^{-1}
\end{array}\right)
$$

After diagonalization of Lagrangian by the transformation

$$
\boldsymbol{\Theta}(x)=\hat{\mathcal{M}} \tilde{\boldsymbol{\Theta}}(x)
$$

we have to rotate the transfer matrix

$$
\hat{\tilde{\mathcal{T}}}=\hat{\mathcal{M}}^{-1} \hat{\mathcal{T}} \hat{\mathcal{M}}
$$

Note that $\hat{\mathcal{M}}=\tau_{1} \hat{\mathcal{M}} \tau_{1}$ and it can be parametrized as follows:

$$
\hat{\mathcal{M}}=\hat{L}^{-1} \hat{M} \hat{L}, \quad \hat{M}=\operatorname{diag}\left\{\hat{M}_{1}, \hat{M}_{2}\right\}
$$

where

$$
\hat{L}=\frac{1}{\sqrt{2}}\left(\begin{array}{cc}
1 & -1 \\
1 & 1
\end{array}\right), \quad \hat{L}^{-1}=\hat{L}^{\mathrm{T}}
$$

Equation (C9) is equivalent to Eq. (17) in the main text after substituting $\hat{M} \rightarrow \hat{M}_{1}$, and $\left(\hat{M}^{-1}\right)^{\mathrm{T}} \rightarrow \hat{M}_{2}$. The symmetry of the transfer matrix in the system with the inversion symmetry ensures that the matrix $\hat{\tilde{\mathcal{T}}}$ has the same structure as $\hat{\mathcal{T}}$, but with different scattering amplitudes:

$$
\hat{\tilde{\mathcal{T}}}=\left(\begin{array}{cc}
\hat{\tilde{T}}-\hat{\hat{R}} \hat{\tilde{T}}^{-1} \hat{\tilde{R}} & \hat{\tilde{R}} \hat{\tilde{T}}^{-1} \\
-\hat{\tilde{T}}^{-1} \hat{\tilde{R}} & \hat{\tilde{T}}^{-1}
\end{array}\right) .
$$

Now, we will find relations between $(\hat{\tilde{R}}, \hat{\tilde{T}})$ and $(\hat{R}, \hat{T})$. 
Let us introduce (auxiliary) rotated transfer matrices

$$
\hat{\mathcal{T}}^{L}=\hat{L} \hat{\mathcal{T}} \hat{L}^{-1}=\frac{1}{2}\left(\begin{array}{ll}
\hat{T}+(1-\hat{R}) \hat{T}^{-1}(1+\hat{R}) & \hat{T}-(1-\hat{R}) \hat{T}^{-1}(1-\hat{R}) \\
\hat{T}-(1+\hat{R}) \hat{T}^{-1}(1+\hat{R}) & \hat{T}+(1+\hat{R}) \hat{T}^{-1}(1-\hat{R})
\end{array}\right)
$$

and

$$
\hat{\tilde{T}}^{L}=\hat{L} \hat{\tilde{T}} \hat{L}^{-1}=\frac{1}{2}\left(\begin{array}{ll}
\hat{\tilde{T}}+(1-\hat{\tilde{R}}) \hat{\tilde{T}}^{-1}(1+\hat{\tilde{R}}) & \hat{\tilde{T}}-(1-\hat{\tilde{R}}) \hat{\tilde{T}}^{-1}(1-\hat{\tilde{R}}) \\
\hat{\tilde{T}}-(1+\hat{\tilde{R}}) \hat{\tilde{T}}^{-1}(1+\hat{\tilde{R}}) & \hat{\tilde{T}}+(1+\hat{\tilde{R}}) \hat{\tilde{T}}^{-1}(1-\hat{\tilde{R}})
\end{array}\right)
$$

The matrix $\hat{\mathcal{T}}^{L}$ can also be expressed via entries of $\hat{\mathcal{T}}^{L}$ :

$$
\hat{\mathcal{T}}^{L}=\hat{M}^{-1} \hat{\mathcal{T}}^{L} \hat{M}=\left(\begin{array}{ll}
\hat{M}_{1}^{-1} \hat{\mathcal{T}}_{11}^{L} \hat{M}_{1} & \hat{M}_{1}^{-1} \hat{\mathcal{T}}_{12}^{L} \hat{M}_{2} \\
\hat{M}_{2}^{-1} \hat{\mathcal{T}}_{21}^{L} \hat{M}_{1} & \hat{M}_{2}^{-1} \hat{\mathcal{T}}_{22}^{L} \hat{M}_{2}
\end{array}\right) .
$$

Using the identity $\hat{L}^{-1} \hat{\tau}_{1} \hat{L}=\hat{\tau}_{3}$, one can prove that the matrix $\hat{\mathcal{T}}^{L}$ obeys the symmetry

$$
\hat{\tau}_{3} \hat{\mathcal{T}}^{L} \hat{\tau}_{3}=\left(\hat{\mathcal{T}}^{L}\right)^{-1},
$$

which means

$$
\left(\begin{array}{lc}
{\left[\hat{\mathcal{T}}_{11}^{L}\right]^{2}-\hat{\mathcal{T}}_{12}^{L} \hat{\mathcal{T}}_{21}^{L}} & -\hat{\mathcal{T}}_{11}^{L} \hat{\mathcal{T}}_{12}^{L}+\hat{\mathcal{T}}_{12}^{L} \hat{\mathcal{T}}_{22}^{L} \\
\hat{\mathcal{T}}_{21}^{L} \hat{\mathcal{T}}_{11}^{L}-\hat{\mathcal{T}}_{22}^{L} \hat{\mathcal{T}}_{21}^{L} & {\left[\hat{\mathcal{T}}_{22}^{L}\right]^{2}-\hat{\mathcal{T}}_{21}^{L} \hat{\mathcal{T}}_{12}^{L}}
\end{array}\right)=1
$$

Thus, we can parametrize $\hat{\mathcal{T}}^{L}$ by, for example, its off-diagonal entries

$$
\hat{\mathcal{T}}_{11}^{L}=\sqrt{1+\hat{\mathcal{T}}_{12}^{L} \hat{\mathcal{T}}_{21}^{L}}, \quad \hat{\mathcal{T}}_{22}^{L}=\sqrt{1+\hat{\mathcal{T}}_{21}^{L} \hat{\mathcal{T}}_{12}^{L}}
$$

A straightforward algebra yields

$$
\begin{aligned}
\hat{\mathcal{T}}^{L} & =\frac{1}{2}\left(\begin{array}{cc}
\hat{\mathcal{T}}_{11}^{L} & -(1-\hat{R}+\hat{T}) \hat{T}^{-1}(1-\hat{R}-\hat{T}) \\
-(1+\hat{R}+\hat{T}) \hat{T}^{-1}(1+\hat{R}-\hat{T}) & \hat{\mathcal{T}}_{22}^{L}
\end{array}\right) \\
& =\left(\begin{array}{cc}
\hat{\mathcal{T}}_{11}^{L} & {\left[(1-\hat{R}+\hat{T})^{-1}-(1-\hat{R}-\hat{T})^{-1}\right]^{-1}} \\
{\left[(1+\hat{R}+\hat{T})^{-1}-(1+\hat{R}-\hat{T})^{-1}\right]^{-1}} & \hat{\mathcal{T}}_{22}^{L}
\end{array}\right) .
\end{aligned}
$$

The same manipulations with Eq. (C14) yield

$$
\hat{\tilde{\mathcal{T}}}^{L}=\left(\begin{array}{cc}
\hat{\tilde{\mathcal{T}}}_{11}^{L} & {\left[(1-\hat{\tilde{R}}+\hat{\tilde{T}})^{-1}-(1-\hat{\tilde{R}}-\hat{\tilde{T}})^{-1}\right]^{-1}} \\
{\left[(1+\hat{\tilde{R}}+\hat{\tilde{T}})^{-1}-(1+\hat{\tilde{R}}-\hat{\tilde{T}})^{-1}\right]^{-1}} & \hat{\tilde{T}}_{22}^{L}
\end{array}\right) .
$$

Combining Eqs. (C14), (C18), and (C20) and inverting matrix entries, we find

$$
\begin{aligned}
& \text { from entries }\{1,2\}:(1-\hat{\tilde{R}}+\hat{\tilde{T}})^{-1}-(1-\hat{\tilde{R}}-\hat{\tilde{T}})^{-1}=\hat{M}_{2}^{-1}\left[(1-\hat{R}+\hat{T})^{-1}-(1-\hat{R}-\hat{T})^{-1}\right] \hat{M}_{1} ; \\
& \text { from entries }\{2,1\}:(1+\hat{\tilde{R}}+\hat{\tilde{T}})^{-1}-(1+\hat{\tilde{R}}-\hat{\tilde{T}})^{-1}=\hat{M}_{1}^{-1}\left[(1+\hat{R}+\hat{T})^{-1}-(1+\hat{R}-\hat{T})^{-1}\right] \hat{M}_{2} .
\end{aligned}
$$

To solve these equations, we parametrize

$$
\hat{S}_{ \pm}=\hat{R} \pm \hat{T}=\frac{1-\hat{\xi}_{ \pm}}{1+\hat{\xi}_{ \pm}} \quad \Rightarrow \quad 1-\hat{S}_{ \pm}=\frac{2 \hat{\xi}_{ \pm}}{1+\hat{\xi}_{ \pm}} \quad \text { and } \quad 1+\hat{S}_{ \pm}=\frac{2}{1+\hat{\xi}_{ \pm}}
$$

and

$$
\hat{\tilde{S}}_{ \pm}=\hat{\tilde{R}} \pm \hat{\tilde{T}}=\frac{1-\hat{\tilde{\xi}}_{ \pm}}{1+\hat{\tilde{\xi}}_{ \pm}} \quad \Rightarrow \quad 1-\hat{\tilde{S}}_{ \pm}=\frac{2 \hat{\xi}_{ \pm}}{1+\hat{\tilde{\xi}}_{ \pm}} \quad \text { and } \quad 1+\hat{\tilde{S}}_{ \pm}=\frac{2}{1+\hat{\tilde{\xi}}_{ \pm}},
$$

and reduce Eqs. (C21) and (C22) to

$$
\begin{gathered}
\hat{\xi}_{+}^{-1}-\hat{\xi}_{-}^{-1}=\hat{M}_{2}^{-1}\left[\hat{\xi}_{+}^{-1}-\hat{\xi}_{-}^{-1}\right] \hat{M}_{1} \\
\hat{\tilde{\xi}}_{+}-\hat{\xi}_{-}=\hat{M}_{1}^{-1}\left[\hat{\xi}_{+}-\hat{\xi}_{-}\right] \hat{M}_{2} .
\end{gathered}
$$


The obvious solution of Eqs. (C25) and (C26), which relates $(\hat{\tilde{R}}, \hat{\tilde{T}})$ and $(\hat{R}, \hat{T})$, reads as

$$
\hat{\tilde{\xi}}_{ \pm}=\hat{M}_{1}^{-1} \hat{\xi}_{ \pm} \hat{M}_{2}
$$

The second solution of Eqs. (C25) and (C26) can be obtained after noticing that they are invariant with respect to transformation $\hat{\tilde{\xi}}_{+} \leftrightarrow-\hat{\tilde{\xi}}_{-}$. Applying this transformation to Eq. (C27) we find

$$
\hat{\tilde{\xi}}_{ \pm}=-\hat{M}_{1}^{-1} \hat{\xi}_{\mp} \hat{M}_{2} .
$$

Note, however, that the solutions (C28) do not satisfy the continuity. Namely, in the noninteracting case, where $\hat{M}_{1}=$ $\hat{M}_{2}$, we require $\hat{\xi}_{ \pm}=\hat{\xi}_{ \pm}$which holds true only for the solution (C27).

\section{APPENDIX D: AUTOCORRELATION FUNCTION $\hat{\Delta}$}

Both weak-scatterer and weak-link perturbations can be written in a unified way [see Eqs. (20) and (21) in the main text]:

$$
\begin{aligned}
& L_{\mathrm{WS}}=\lambda \cos [\Phi(T=1)], \\
& L_{\mathrm{WL}}=t \cos [\Phi(T=0)],
\end{aligned}
$$

where the field $\Phi(T)$ is the difference between two incoming chiral fields of the first channel for the conducting phase $(T=$ 1) and for the insulating phase $(T=0)$ :

$$
\begin{aligned}
\Phi(T)= & {\left[\theta_{\mathrm{R}}^{(1)}(x=-0, t)-\theta_{\mathrm{L}}^{(1)}(x=+0, t)\right] \text { at } } \\
& \left\{\begin{array}{lll}
T=1, & R=0 & \text { for CP, } \\
T=0, & R=1 & \text { for IP. }
\end{array}\right.
\end{aligned}
$$

The notation in Eq. (D3) stresses that the field $\Phi(T)$ and its autocorrelation function depend on boundary conditions including, of course, boundary conditions in the LL channel. In particular, one can restore formulas for the same perturbations introduced in Ref. [35] by using the matching conditions

$$
\begin{gathered}
\theta_{\mathrm{R}, \mathrm{L}}^{(1)}(x=-0, t)=\theta_{\mathrm{R}, \mathrm{L}}^{(1)}(x=+0, t) \quad \text { in the CP, } \\
\theta_{\mathrm{R}}^{(1)}(x= \pm 0, t)=\theta_{\mathrm{L}}^{(1)}(x= \pm 0, t) \quad \text { in the IP. }
\end{gathered}
$$

As discussed in the main text, the RG equations are governed by the retarded component of the Green's function $\mathcal{G}(t-$ $\left.t^{\prime} ; T\right)=-i\left\langle\Phi(t ; T) \Phi\left(t^{\prime} ; T\right)\right\rangle$ :

$$
\mathcal{G}\left(\omega_{+} ; T\right) \equiv g_{0}[\hat{\Delta}(T)]_{11} .
$$

Here, we have defined the $N \times N$ matrix $\hat{\Delta}(T)$ which depends on the phase (either the CP or the IP) and can be found by using the Green's functions introduced in Appendix A:

$$
\hat{\Delta}=\frac{1}{g_{0}}\left[\hat{G}_{--}^{R R}+\hat{G}_{++}^{L L}-\hat{G}_{-+}^{R L}-\hat{G}_{+-}^{L R}\right] ;
$$

superscripts are related to chirality indices. Using Eq. (B15) we arrive at

$$
\hat{\Delta}=\frac{1}{2} \operatorname{tr}_{\mathrm{ch}} \hat{\mathcal{M}}\left(\begin{array}{cc}
1 & \hat{\tilde{S}}_{-} \\
\hat{\tilde{S}}_{-} & 1
\end{array}\right) \hat{\mathcal{M}}^{\mathrm{T}}, \quad \hat{\tilde{S}}_{-}=\hat{\tilde{R}}-\hat{\tilde{T}} .
$$

Trace $\operatorname{tr}_{\mathrm{ch}}$ is calculated over the chiral space of right/left movers; expanding it in Eq. (D8) we find

$$
\hat{\Delta}=\frac{1}{2}\left[\hat{M}_{1}\left(1-\hat{\tilde{S}}_{-}\right) \hat{M}_{1}^{\mathrm{T}}+\hat{M}_{2}\left(1+\hat{\tilde{S}}_{-}\right) \hat{M}_{2}^{\mathrm{T}}\right]
$$

Now we (a) use Eqs. (C24) and (C27) to express $\hat{\tilde{S}}_{-}$in terms of $\hat{\xi}_{-}$and matrices $\hat{M}_{1,2}$; and (b) simplify Eq. (D9) to

$$
\begin{aligned}
\hat{\Delta} & =\left[\hat{\xi}_{-}^{-1}+\hat{\delta}^{-1}\right]^{-1} \hat{M}_{2} \hat{M}_{1}^{\mathrm{T}}+\left[\hat{\xi}_{-}+\hat{\delta}\right]^{-1} \hat{M}_{1} \hat{M}_{2}^{\mathrm{T}}, \\
\hat{\delta} & \equiv \hat{M}_{1} \hat{M}_{2}^{-1} .
\end{aligned}
$$

The symmetry Eq. (13) in the main text implies

$$
\hat{M}_{1} \hat{M}_{2}^{\mathrm{T}}=1 .
$$

Taking this into account (and skipping subscripts of $\hat{\xi}_{-}$and $\hat{M}_{1}$ ), we reduce Eqs. (D9) and (D10) to Eqs. (25) and (26) of the main text.

\section{APPENDIX E: EXAMPLE: SCALING EXPONENTS IN THE CASES OF A SINGLE LL}

The single-channel Lagrangian for bosons $\hat{\Theta}^{\mathrm{T}}=\left(\theta_{R}, \theta_{L}\right)$ moving right $\theta_{R}$ and left $\theta_{L}$ with unperturbed velocity $v_{\mathrm{F}}$ is given by

$$
L_{1}=\frac{1}{4 \pi}\left(\theta_{R}, \theta_{L}\right)\left[\hat{\tau}_{3} \partial_{t}+\left(v_{\mathrm{F}}+\hat{V}_{1}\right) \partial_{x}\right] \partial_{x}\left(\begin{array}{c}
\theta_{R} \\
\theta_{L}
\end{array}\right) ;
$$

the subscript " 1 " is used in this section to emphasize that only one channel is studied. Here, we have defined the interaction matrix

$$
\hat{V}=v_{\mathrm{F}}\left(\begin{array}{cc}
g_{4} & -g_{2} \\
-g_{2} & g_{4}
\end{array}\right)
$$

using standard " $g$-ology" notations for intermode interactions $g_{2}$ and for intramodes ones $g_{4}$. Let us introduce a matrix of hyperbolic rotations

$$
\begin{aligned}
\hat{\tau}(\varphi) & =\left(\begin{array}{cc}
\cosh \varphi & \sinh \varphi \\
\sinh \varphi & \cosh \varphi
\end{array}\right), \quad \hat{\tau}(\varphi) \hat{\tau}_{3} \hat{\tau}(\varphi)=\hat{\tau}_{3}, \\
\hat{\tau}\left(\varphi_{1}\right) \hat{\tau}\left(\varphi_{2}\right) & =\hat{\tau}\left(\varphi_{1}+\varphi_{2}\right),
\end{aligned}
$$

which parametrizes $1+\hat{V} / v_{\mathrm{F}}$ :

$1+\frac{1}{v_{\mathrm{F}}} \hat{V}=\left(\begin{array}{cc}1+g_{4} & -g_{2} \\ -g_{2} & 1+g_{4}\end{array}\right)=\sqrt{\left(1+g_{4}\right)^{2}-g_{2}^{2}} \hat{\tau}^{2}\left(-\varphi_{0}\right)$,

$\tanh 2 \varphi_{0}=\frac{g_{2}}{1+g_{4}}$.

It follows from Eqs. (E3) and (E4) that one can diagonalize the Lagrangian $L_{1}$ with the help of a transformation

$$
\left(\begin{array}{l}
\theta_{R} \\
\theta_{L}
\end{array}\right)=\hat{\mathcal{M}}_{1}\left(\begin{array}{c}
\tilde{\theta}_{R} \\
\tilde{\theta}_{L}
\end{array}\right), \quad \hat{\mathcal{M}}_{1} \equiv \hat{\tau}\left(\varphi_{0}\right)
$$

The Lagrangian for new chiral bosonic fields reads as

$$
\begin{aligned}
\tilde{L}_{1} & =\frac{1}{4 \pi}\left(\tilde{\theta}_{R}, \tilde{\theta}_{L}\right)\left[\hat{\tau}_{3} \partial_{t}+u \partial_{x}\right] \partial_{x}\left(\begin{array}{c}
\tilde{\theta}_{R} \\
\tilde{\theta}_{L}
\end{array}\right), \\
u & =v_{F} \sqrt{\left(1+g_{4}\right)^{2}-g_{2}^{2}} .
\end{aligned}
$$


Following notations used in the main text [Eq. (17)], we rewrite the matrix $\mathcal{M}_{1}$ as follows:

$$
\begin{aligned}
\hat{\mathcal{M}}_{1} & =\left(\begin{array}{ll}
K^{-1 / 2}+K^{1 / 2} & K^{-1 / 2}-K^{1 / 2} \\
K^{-1 / 2}-K^{1 / 2} & K^{-1 / 2}+K^{1 / 2}
\end{array}\right) \\
& =\frac{1-\tau_{1}}{2} M_{1}+\frac{1+\tau_{1}}{2} M_{1}^{-1}
\end{aligned}
$$

with

$$
M_{1} \equiv K^{1 / 2}, \quad K \equiv e^{-2 \phi_{0}} ;
$$

here, $K$ is the usual Luttinger parameter. We note that the "matrix" $M_{1}$ and, correspondingly, $\delta_{1}=M_{1}^{2}$ are scalars because we consider only one channel. Inserting this expression for $\delta_{1}$ into Eqs. (30) and (31) of the main text we find the scaling dimensions for the single LL:

$$
\Delta_{\mathrm{WS}}=\delta=K, \quad \Delta_{\mathrm{WL}}=\delta^{-1}=K^{-1} .
$$

\section{APPENDIX F: DUALITY OF MATRIX ELEMENTS}

Let us consider $N \times N$ symmetric matrix and write it as a block matrix

$$
\left(\begin{array}{cc}
a_{0} & \psi^{\mathrm{T}} \\
\psi & \hat{b}
\end{array}\right)
$$

where $a_{0}$ is scalar, $\psi$ is $(N-1)$-dimensional vector, and $\hat{b}$ is $(N-1) \times(N-1)$ symmetric matrix. Its inversion is given by

$$
\begin{aligned}
\left(\begin{array}{cc}
a_{0} & \psi^{\mathrm{T}} \\
\psi & \hat{b}
\end{array}\right)^{-1} & =\left(\begin{array}{cc}
A & -A\left[\hat{b}^{-1} \psi\right]^{\mathrm{T}} \\
-A \hat{b}^{-1} \psi & {\left[\hat{b}-\psi \otimes \psi^{\mathrm{T}} / a_{0}\right]^{-1}}
\end{array}\right), \\
A & =\left(a_{0}-\psi^{\mathrm{T}} \hat{b}^{-1} \psi\right)^{-1} .
\end{aligned}
$$

Our goal is to find matrix entries

$$
\Delta_{\mathrm{WS}}=\lim _{\xi \rightarrow \infty}\left[\frac{1}{\hat{\delta}^{-1}+\hat{\xi}^{-1}}\right]_{11}, \quad \Delta_{\mathrm{WL}}=\lim _{\xi \rightarrow 0}\left[\frac{1}{\hat{\delta}+\hat{\xi}}\right]_{11},
$$

where $\xi=\hat{\xi}_{11}, \hat{\delta}=\hat{M} \hat{M}^{\mathrm{T}}$, and the matrices $\hat{\delta}$ and $\hat{\xi}$ can be written in the decomposition (F1) as follows:

$$
\hat{\delta}=\left(\begin{array}{cc}
\delta & \psi^{\mathrm{T}} \\
\psi & \hat{\delta}_{\mathrm{e}}
\end{array}\right), \quad \hat{\xi}=\left(\begin{array}{cc}
\xi & 0 \\
0 & \hat{\zeta}
\end{array}\right)
$$

[see Eqs. (26) and (27) in the main text].

Using Eq. (F2), we find

$$
\Delta_{\mathrm{WL}}=\left(\begin{array}{cc}
\delta & \psi^{\mathrm{T}} \\
\psi & \hat{\delta}_{\mathrm{e}}+\hat{\zeta}
\end{array}\right)_{11}^{-1}=\left[\delta-\psi^{\mathrm{T}}\left(\hat{\delta}_{\mathrm{e}}+\hat{\zeta}\right)^{-1} \psi\right]^{-1} .
$$

To calculate $\Delta_{\mathrm{WS}}$, let us use the identity

$$
\left(\hat{\alpha}^{-1}+\hat{\beta}^{-1}\right)^{-1}=\hat{\alpha}(\hat{\alpha}+\hat{\beta})^{-1} \hat{\beta}
$$

which holds true for nonsingular matrices $\hat{\alpha}$ and $\hat{\beta}$ and allows us to write the formula for $\Delta_{\mathrm{WS}}$ as follows:

$$
\Delta_{\mathrm{WS}}=\lim _{\xi \rightarrow \infty}\left[\hat{\delta}(\hat{\delta}+\hat{\xi})^{-1} \hat{\xi}\right]_{11}=\left[\hat{\delta} \lim _{\xi \rightarrow \infty}\left\{\xi(\hat{\delta}+\hat{\xi})^{-1}\right\}\right]_{11} .
$$

The limit $\xi \rightarrow \infty$ is easily found from the inversion $(\mathrm{F} 2)^{(\mathrm{F} 7)}$

$$
\begin{array}{ll}
\lim _{\xi \rightarrow \infty}\left\{\xi(\hat{\delta}+\hat{\xi})^{-1}\right\} & \\
=\left(\begin{array}{cc}
1 & -\left[\left(\hat{\delta}_{\mathrm{e}}+\hat{\zeta}\right)^{-1} \psi\right]^{\mathrm{T}} \\
-\left(\hat{\delta}_{\mathrm{e}}+\hat{\zeta}\right)^{-1} \psi & -\psi \otimes \psi^{\mathrm{T}}
\end{array}\right) .
\end{array}
$$

Inserting Eq. (F8) into (F7), we find

$$
\Delta_{\mathrm{WS}}=\delta-\psi^{\mathrm{T}}\left(\hat{\delta}_{\mathrm{e}}+\hat{\zeta}\right)^{-1} \psi \Rightarrow \Delta_{\mathrm{WS}}=\Delta_{\mathrm{WL}}^{-1}
$$

[1] S. Tomonaga, Prog. Theor. Phys. 5, 544 (1950).

[2] J. M. Luttinger, J. Math. Phys. 4, 1154 (1963).

[3] F. D. M. Haldane, J. Phys. C: Solid State Phys. 14, 2585 (1981).

[4] T. Giamarchi, Quantum Physics in One Dimension (Clarendon, London, 2004).

[5] M. Bockrath, D. H. Cobden, J. Lu, A. G. Rinzler, R. E. Smalley, L. Balents, and P. L. McEuen, Nature (London) 397, 598 (1999).

[6] C. L. Kane and M. P. A. Fisher, Phys. Rev. B 51, 13449 (1995).

[7] C. L. Kane, R. Mukhopadhyay, and T. C. Lubensky, Phys. Rev. Lett. 88, 036401 (2002).

[8] C.-Y. Hou, E.-A. Kim, and C. Chamon, Phys. Rev. Lett. 102, 076602 (2009).

[9] J. C. Y. Teo and C. L. Kane, Phys. Rev. B 79, 235321 (2009).

[10] I. Safi and H. Saleur, Phys. Rev. Lett. 93, 126602 (2004).

[11] M. Kindermann and Y. V. Nazarov, Phys. Rev. Lett. 91, 136802 (2003).

[12] I. Bloch, J. Dalibard, and W. Zwerger, Rev. Mod. Phys. 80, 885 (2008).

[13] S. Q. Shen, Topological Insulators (Springer, Berlin, 2012).
[14] M. A. Cazalilla, R. Citro, T. Giamarchi, E. Orignac, and M. Rigol, Rev. Mod. Phys. 83, 1405 (2011).

[15] B. L. Altshuler, I. L. Aleiner, and V. I. Yudson, Phys. Rev. Lett. 111, 086401 (2013).

[16] A. O. Gogolin, A. A. Nersesyan, and T. A. M, Bosonization and Strongly Correlated Systems (Cambridge University Press, Cambridge, 2004).

[17] M. A. Cazalilla, J. Phys. B: At., Mol. Opt. Phys. 37, S1 (2004).

[18] Z. Yao, H. W. C. Postma, L. Balents, and C. Dekker, Nature (London) 402, 273 (1999).

[19] H. Ishii et al., Nature (London) 426, 540 (2003).

[20] J. Lee, S. Eggert, H. Kim, S. J. Kahng, H. Shinohara, and Y. Kuk, Phys. Rev. Lett. 93, 166403 (2004).

[21] O. M. Auslaender, A. Yacoby, R. de Picciotto, K. W. Baldwin, L. N. Pfeiffer, and K. W. West, Science 295, 825 (2002).

[22] E. Slot, M. A. Holst, H. S. J. van der Zant, and S. V. ZaitsevZotov, Phys. Rev. Lett. 93, 176602 (2004).

[23] E. Levy, A. Tsukernik, M. Karpovski, A. Palevski, B. Dwir, E. Pelucchi, A. Rudra, E. Kapon, and Y. Oreg, Phys. Rev. Lett. 97, 196802 (2006). 
[24] L. Venkataraman, Y. S. Hong, and P. Kim, Phys. Rev. Lett. 96, 076601 (2006).

[25] C. L. Kane and M. P. A. Fisher, Phys. Rev. Lett. 68, 1220 (1992).

[26] C. L. Kane and M. P. A. Fisher, Phys. Rev. B 46, 15233 (1992).

[27] K. A. Matveev, D. Yue, and L. I. Glazman, Phys. Rev. Lett. 71, 3351 (1993).

[28] A. Furusaki and N. Nagaosa, Phys. Rev. B 47, 4631 (1993).

[29] A. Furusaki, Phys. Rev. B 56, 9352 (1997).

[30] S. Eggert and I. Affleck, Phys. Rev. Lett. 75, 934 (1995).

[31] M. Fabrizio and A. O. Gogolin, Phys. Rev. B 51, 17827 (1995).

[32] P. San-Jose, F. Guinea, and T. Martin, Phys. Rev. B 72, 165427 (2005).

[33] A. Galda, I. V. Yurkevich, and I. V. Lerner, Phys. Rev. B 83, R041106 (2011).

[34] A. Galda, I. V. Yurkevich, and I. V. Lerner, Europhys. Lett. 93, 17009 (2011).
[35] I. V. Yurkevich, A. Galda, O. M. Yevtushenko, and I. V. Lerner, Phys. Rev. Lett. 110, 136405 (2013).

[36] I. V. Yurkevich, Europhys. Lett. 104, 37004 (2013).

[37] We note that the Lagrangian of the system with the inversion symmetry can always be diagonalized by using the matrix $\hat{\mathcal{M}}$ (see Appendix A for details).

[38] The scattering state representation is described in Appendix B.

[39] The explicit relation between $(\hat{\tilde{R}}, \hat{\tilde{T}})$ and $(\hat{R}, \hat{T})$ is given in Appendix C.

[40] This step is described in Appendix D.

[41] Details can be found in Appendix E.

[42] The mathematical proof of this conclusion is given in Appendix F.

[43] A. Altland, Y. Gefen, and B. Rosenow, Phys. Rev. Lett. 108, 136401 (2012).

[44] N. Sedlmayr, J. Ohst, I. Affleck, J. Sirker, and S. Eggert, Phys. Rev. B 86, 121302 (2012). 